\title{
Ceratoplastias penetrantes realizadas na Fundação Altino Ventura: revisão dos resultados e complicações
}

\author{
Penetrating keratoplasties performed at the Fundação Altino Ventura: review of results \\ and complications
}

Daniela Endriss ${ }^{1}$

Fernando Cunha ${ }^{2}$

Marco Pólo Ribeiro ${ }^{3}$

Jana Toscano4

\section{RE S U M O}

Objetivo: Identificar as principais complicações e causas de insucesso das ceratoplastias penetrantes realizadas na Fundação Altino Ventura, RecifePE. Métodos: Revisaram-se os prontuários médicos de 205 pacientes, submetidos a ceratoplastia penetrante na Fundação Altino Ventura, no período de janeiro de 1999 a dezembro de 2000 , com tempo de seguimento pós-operatório maior ou igual a seis meses. Avaliaram-se dados demográficos e relacionados aos períodos pré, per e pós-operatório. Resultados: A média de idade dos pacientes foi de 48,4 $( \pm 22,1)$ anos, sendo $108(52,7 \%)$ do sexo feminino. As principais indicações do transplante penetrante de córnea foram ceratocone (49 casos, $23,9 \%$ ), ceratopatia bolhosa pósfacectomia ( 48 casos, $23,4 \%$ ) e distrofias ( 24 casos, $11,7 \%$ ). A complicação pós-operatória mais freqüentemente encontrada foi glaucoma em 65 casos $(31,7 \%)$. Cinqüenta pacientes $(24,4 \%)$ apresentaram sinais clínicos de rejeição do botão, havendo evolução para insucesso do transplante em 13 $(26,0 \%)$ destes casos. Observou-se que $16,0 \%(n=8)$ dos pacientes com rejeição foram submetidos a três ou mais procedimentos cirúrgicos, diferença estatisticamente significante em relação ao grupo que não desenvolveu rejeição $(3,2 \%, \mathrm{n}=5)(\mathrm{p}<0,05)$. Quarenta e cinco $(22,0 \%)$ transplantes realizados evoluíram com opacificação total do botão doador. Conclusão: Os fatores de risco para falência do transplante de córnea identificados neste estudo concordam com os da literatura mundial. O glaucoma foi a principal complicação pós-operatória identificada e o principal fator predisponente à rejeição e falência do transplante.

Descritores:Ceratoplastia penetrante/efeitos adversos;Complicações pós-operatórias; Resultadodetratamento

Trabalho realizado na Fundação Altino Ventura com apo do Hospital de Olhos de Pernambuco, em Recife-PE.

${ }^{1}$ Médica, Fellow do Departamento de Córnea da Fundação Altino Ventura.

${ }^{2}$ Médico, Staff do Departamento de Córnea da Fundação Altino Ventura e Hospital de Olhos de Pernambuco.

${ }^{3}$ Médico, aluno do primeiro ano do Curso de Especialização em Oftalmologia da Fundação Altino Ventura.

${ }^{4}$ Médica, aluna do terceiro ano do Curso de Especialização em Oftalmologia da Fundação Altino Ventura.

Endereço para correspondência: Rua do Progresso, 317/604 Boa Vista - Recife (PE) - Brasil CEP 50070-020 E-mail: danielaendriss@yahoo.com.br

Recebido para publicação em 25.02.2002 Aceito para publicação em 07.10.2002

Nota Editorial: Pela análise deste trabalho e por sua anuência na divulgação desta nota, agradecemos ao Dr. Marcelo Luís Occhiutto. \begin{tabular}{l}
\hline I N T R O D C Ã O \\
\hline A ceratoplastia penetrante é o procedimento de maior sucesso entre os \\
transplantes teciduais em humanos e tem sido o mais realizado na atualida- \\
de ${ }^{(1-3)}$. O sucesso desse procedimento cirúrgico tem sido atribuído ao fato da \\
córnea ser avascular e aos privilégios imunológicos desta estrutura. Avan- \\
ços na técnica cirúrgica, equipamento, materiais, métodos de preservação da \\
córnea doadora e manejo pós-operatório também têm contribuído para o \\
sucesso dos transplantes de córnea \\
O prognóstico dos transplantes de córnea é de natureza multifatorial ${ }^{(6)}$. \\
Apesar dos progressos evidentes, a evolução pós-operatória da ceratoplas- \\
tia penetrante depende de fatores prognósticos relacionados ao quadro \\
clínico da doença ocular que motiva a indicação cirúrgica. O reconhecimento
\end{tabular} 
destes fatores torna-se essencial para minimizar as taxas de insucesso, promovendo melhor resultado funcional visual para o paciente ${ }^{(1)}$. A maioria dos cirurgiões de córnea reconhecem que há muitas complicações pós-operatórias inesperadas, observadas em pacientes com poucos fatores de risco e submetidos a transplante de córnea sem intercorrências. Deve ser dado ênfase na prevenção, reconhecimento precoce e tratamento das complicações, através de avaliações pós-operatórias freqüentes e cuidados de rotina efetivos ${ }^{(2)}$.

Apesar de ter evoluído ao longo dos anos, o controle efetivo da rejeição no transplante de córnea é ainda insuficientemente compreendido. Enquanto o progresso nas pesquisas em imunossupressão e imunomodulação visam superar os efeitos da rejeição no transplante de córnea, atualmente, a estratégia mais efetiva é reconhecer a rejeição precocemente e tratá-la agressivamente ${ }^{(2)}$. Vários estudos foram realizados para identificar os fatores predisponentes da rejeição em transplante de córnea, entre estes estão a vascularização corneana, o diâmetro do botão transplantado, a existência de um transplante prévio, a experiência do cirurgião, e o aumento da pressão intra-ocular no pós-operatório ${ }^{(3,6-8)}$.

Motivados pela escassez de dados na literatura sobre as ceratoplastias penetrantes realizadas na região nordeste do Brasil, além da necessidade em conhecer os resultados finais deste procedimento, os autores realizaram este estudo com o objetivo de avaliar as principais indicações, complicações pós-operatórias e causas de insucesso das ceratoplastias penetrantes realizadas na Fundação Altino Ventura, um centro de referência em Recife - PE.

\section{MÉ TODOS}

Realizou-se um estudo retrospectivo analisando-se os prontuários médicos dos pacientes submetidos à ceratoplastia penetrante na Fundação Altino Ventura, no período de janeiro de 1999 a dezembro de 2000. A avaliação incluiu o exame até a última consulta agendada ou até a perda do seguimento. Excluíram-se os casos de transplantes tectônicos, lamelares ou com tempo mínimo de acompanhamento pós-operatório inferior a seis meses. Cinco pacientes foram submetidos a um segundo transplante de córnea, porém os dados referentes à reoperação foram excluídos da amostra para assegurar independência na análise.

Analisou-se a amostra de 205 pacientes quanto aos dados demográficos (idade e sexo) e dados relacionados aos períodos pré, per e pós-operatório, que incluíram: indicação do transplante, acuidade visual pré-operatória, antecedentes oftalmológicos, idade do botão doador, tipo de cirurgia realizada (transplante como procedimento único ou combinado), acuidade visual final com melhor correção, complicações e ocorrência de rejeição e/ou falência do botão.

Diagnosticou-se rejeição do transplante nos casos de aumento da inflamação associado a sinais corneanos de edema, vascularização, precipitados ceráticos, linha de Khodadoust, ou infiltrados subepiteliais ocorrendo após as primeiras duas semanas pós-operatórias. Definiu-se como falência do transplante os casos de edema estromal persistente por um período superior a um mês de tratamento intenso com esteróide tópico, ou vascularização do botão. Diagnosticou-se falência primária do enxerto a ausência de transparência do botão doador desde o primeiro dia pós-operatório, e falência tardia como perda da transparência em córneas doadoras previamente claras.

Considerou-se glaucoma como pressão intra-ocular acima de $21 \mathrm{mmHg}$ por pelo menos dois meses, requerendo uso de medicação hipotensora.

Os residentes do terceiro ano realizaram as cirurgias, supervisionados por um staff do Departamento de Córnea. Preparou-se a córnea receptora com um diâmetro variando de 7,25 a 8,25 mm, e o botão doador com um diâmetro 0,25 a 0,50 mm maior que o leito receptor, através da trepanação da superfície endotelial. Para a sutura, utilizou-se fio mononylon $10.0 \mathrm{com}$ 16 pontos interrompidos. Realizaram-se outros procedimentos como facectomia, implante de lente intra-ocular (LIO), vitrectomia anterior, pupiloplastia ou uma combinação destes quando clinicamente indicado. As medicações utilizadas de rotina no pós-operatório são ofloxacina colírio seis vezes ao dia, e acetato de prednisolona $1 \%$ colírio, inicialmente de $2 / 2$ horas, com diminuição gradativa conforme cada evolução.

Categorizou-se a acuidade visual (AV) final com correção em: (1) 20/20 a 20/40; (2) 20/50 a 20/150; (3) 20/200 a conta dedos (CD); (4) movimento de mãos (MM) a percepção luminosa (PL) e (5) sem percepção luminosa (SPL).

Utilizou-se para análise estatística o método do qui-quadrado de aderência, sendo considerado o valor de $\mathrm{p}<0,05$ como estatisticamente significante.

RESULTADOS

A idade dos pacientes estudados variou de cinco a 89 anos, média de 48,4 $\pm 22,1$ anos, sendo $108(52,7 \%)$ do sexo feminino e $97(47,3 \%)$ do masculino. No período pré-operatório, 138 pacientes $(67,3 \%)$ apresentaram acuidade visual entre 20/200 e conta dedos (Gráfico 1). A idade do botão doador variou de seis a 74 anos, média de 40,1 $\pm 16,1$ anos. Nos antecedentes oftalmológicos, identificou-se glaucoma em 32 pacientes $(15,6 \%)$, e em $12(37,5 \%)$ destes havia história pregressa de cirurgia antiglaucomatosa. A pressão intra-ocular encontrava-se controlada na época da realização do transplante de córnea. As principais indicações do transplante penetrante de córnea foram ceratocone (49 casos, 23,9\%) e ceratopatia bolhosa pósfacectomia (48 casos, 23,4\%) (Tabela 1). Dos pacientes com ceratopatia bolhosa pós-facectomia, 14 eram afácicos $(29,2 \%)$ e $34(70,8 \%)$ eram pseudo-fácicos. Avaliando-se estes pacientes, observou-se que 46 casos $(95,8 \%)$ vieram encaminhados de outros serviços e dois pacientes $(4,2 \%)$ desenvolveram ceratopatia bolhosa pós-facectomia realizada na Fundação Altino Ventura. Dos 24 casos de distrofias, 21 (87,5\%) eram distrofia de Fuchs. 


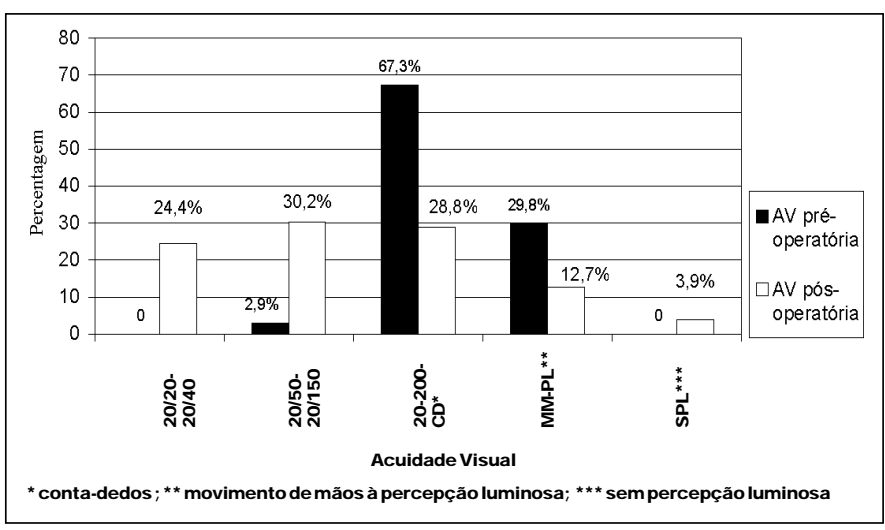

Gráfico 1. Distribuição dos pacientes submetidos à ceratoplastia penetrante segundoa acuidade visual (AV) prée és-operatória. FundaçãoAltinoVentura,2001

Tabela 1.Distribuiçãodos pacientes submetidos à ceratoplastia
penetrantequantoâindicaçãocinúrgica. FundaçãoAltinoVentura, penetrantequantoàindicaçãocinúrg

\begin{tabular}{lcc|} 
Indicações & $\mathbf{N}$ & $\%$ \\
Ceratocone & 49 & 23,9 \\
Ceratopatiabolhosa pós-facectomia & 48 & 23,4 \\
Distrofias & 24 & 11,7 \\
Ceratiteinfecciosa & 22 & 10,7 \\
Trauma & 19 & 9,3 \\
Leucomanainfância & 14 & 6,8 \\
Retransplante & 12 & 5,9 \\
Ceratiteintersticial & 11 & 5,4 \\
Outros* & 6 & 2,9 \\
Total & 205 & 100,0 \\
*leucomapósqueimaduraquímica(2),leucomapóstracoma(2),ceratopatia \\
emfaixa(2) & \multicolumn{3}{|}{} \\
\hline \multicolumn{2}{|c}{} \\
\hline
\end{tabular}

Quanto ao tipo de cirurgia realizada, 152 pacientes $(74,1 \%)$ submeteram-se à ceratoplastia penetrante como procedimento simples, $40(19,5 \%)$ submeteram-se a um outro procedimento associado à ceratoplastia e $13(6,4 \%)$ a dois ou mais procedimentos além da ceratoplastia. Dentre os procedimentos associados incluíram-se: facectomia com implante de LIO (32 casos, 15,6\%), facectomia sem LIO (quatro casos, 2,0\%), fixação escleral da LIO (12 casos, $5,9 \%$ ), vitrectomia anterior (11 casos, $5,4 \%$ ) e pupiloplastia (sete casos, $3,4 \%$ ).

A AV final foi de $20 / 40$ ou melhor em 50 pacientes $(24,4 \%)$ (Gráfico 2). Entre os oito pacientes (3,9\%) que evoluíram com AV final SPL, sete $(87,5 \%)$ apresentaram glaucoma no pósoperatório e três $(37,5 \%)$ descolamento de retina.

O tempo médio de acompanhamento pós-operatório variou de seis a 29 meses, média de $13,5 \pm 5,8$ meses. A complicação mais freqüente encontrada no pós-operatório foi glaucoma acometendo 70 pacientes $(34,1 \%)$ (Tabela 2$)$. Treze pacientes $(6,3 \%)$ apresentaram mais de uma complicação pós-operatória. Foi necessária a realização de cirurgia antiglaucomatosa (trabeculectomia e/ou implante de tubo de drenagem) em 28 (40,0\%) do total de 70 pacientes com glaucoma no pós-operatório.

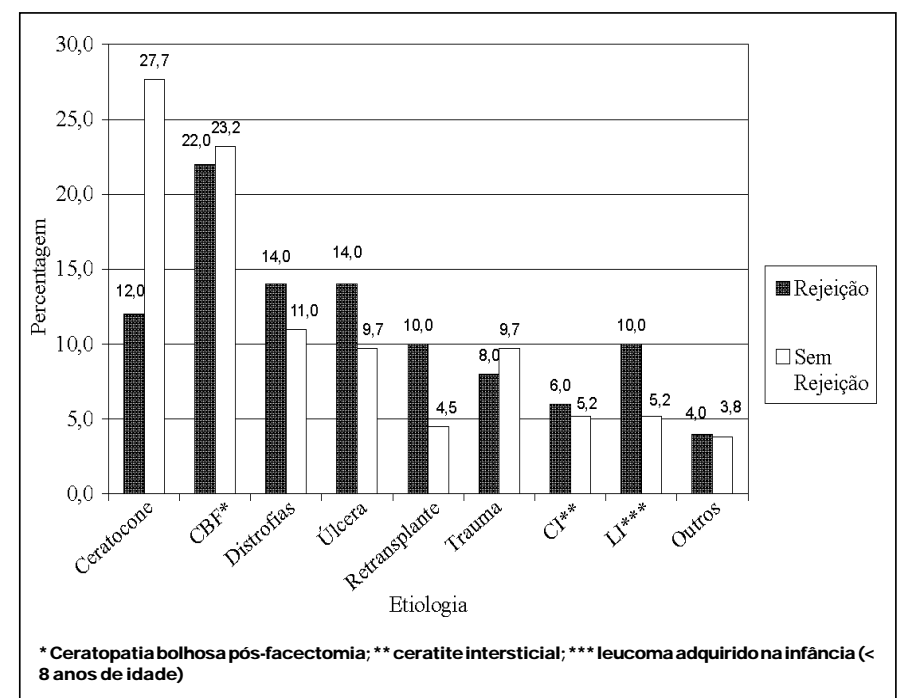

Gráfico 2. Distribuição dos pacientes submetidos à ceratoplastia penetrante segundo à etiologia eà ocorrência ou não de rejeição. FundaçãoAltinoVentura, 2001

\begin{tabular}{|c|c|c|}
\hline Complicações & $N(N=205)$ & $\%$ \\
\hline Glaucoma & б & 31,7 \\
\hline Rejeição & 50 & 24,4 \\
\hline Falência & 45 & $\mathbf{2 2 , 0}$ \\
\hline Ceratiteinfecciosa & 13 & 6,3 \\
\hline Descolamentoderetina & 6 & 2,9 \\
\hline Catarata & 6 & 2,9 \\
\hline Defeitoepitelialpersistente & 5 & 2,4 \\
\hline Outros* & 4 & 2,0 \\
\hline
\end{tabular}

Houve opacificação total do botão em dez $(76,9 \%)$ dos treze pacientes que desenvolveram úlcera infecciosa. Dentre os seis pacientes que evoluíram com descolamento de retina no pósoperatório, quatro $(66,7 \%)$ possuíam etiologia traumática.

Cinqüenta pacientes $(24,4 \%)$ apresentaram algum sinal clínico de rejeição do botão, havendo evolução para insucesso do transplante em $13(26,0 \%)$ destes casos. No grupo dos pacientes que desenvolveram rejeição do botão, observou-se maior freqüência de fatores de risco pré-operatórios $(44,0 \%, \mathrm{n}=22) \mathrm{e}$ complicações pós-operatórias $(58,0 \%, \mathrm{n}=29)$, em relação ao grupo que não apresentou sinais de rejeição $(32,9 \%, n=51 \mathrm{e}$ $45,2 \%, \mathrm{n}=70$ respectivamente) (Tabela 3 ). O tempo médio de ocorrência de rejeição foi de $6,8 \pm 5,3$ meses, variando de 15 dias a 19 meses após o transplante, sendo 49 casos $(98,0 \%)$ de rejeição endotelial e um caso $(2,0 \%)$ de rejeição epitelial. O gráfico 2 mostra a distribuição dos pacientes que desenvolveram ou não rejeição em relação à etiologia. Quanto ao tipo de cirurgia realizada, observou-se que $16,0 \%(\mathrm{n}=8)$ dos pacientes com rejeição foram submetidos a três ou mais procedimentos, 


\begin{tabular}{|c|c|c|c|c|c|c|c|}
\hline \multicolumn{2}{|c|}{ Fatores de Risco/Complicações } & \multicolumn{2}{|c|}{$\begin{array}{c}\text { Rejeição(n=50) } \\
\text { N } \%\end{array}$} & \multicolumn{2}{|c|}{$\begin{array}{c}\text { SemRejeição(n=155) } \\
\mathbf{N}\end{array}$} & \multicolumn{2}{|c|}{ Total $(n=205)$} \\
\hline \multirow[t]{3}{*}{ Pré-operatórias } & Glaucoma & 10 & 20,0 & $\mathbf{2}$ & 14,2 & 32 & 15,6 \\
\hline & Sinéquias/Neovasos & 2 & 4,0 & 2 & 1,3 & 4 & 2,0 \\
\hline & Recobrimentoconjuntival & 3 & 6,0 & 14 & 9,0 & 17 & 8,3 \\
\hline \multirow[t]{4}{*}{ Pós-operatórias } & Glaucoma & 18 & 36,0 & 47 & 30,3 & $\mathbf{6}$ & 31,7 \\
\hline & Ceratiteinfecciosa & 4 & 8,0 & 9 & 5,8 & 13 & 6,3 \\
\hline & Descolamentoderetina & 2 & 4,0 & 4 & 2,6 & 6 & 2,9 \\
\hline & Catarata & 1 & 2,0 & 5 & 3,2 & 6 & 2,9 \\
\hline
\end{tabular}

diferença estatisticamente significante em relação ao grupo que não desenvolveu rejeição $(3,2 \%, n=5)(p<0,05)$. A AV final mostrou-se inferior no grupo com rejeição em relação ao grupo que não desenvolveu rejeição, no entanto, esta diferença não foi estatisticamente significativa $(\mathrm{p}>0,05)$.

Quarenta e cinco $(22,0 \%)$ transplantes realizados evoluíram com opacificação total do botão doador, caracterizando-se falência primária em 15 destes $(33,3 \%)$. Identificou-se falência tardia em $17(37,8 \%)$ casos, onze destes $(64,7 \%)$ estavam relacionados a glaucoma no pós-operatório. Em 13 pacientes $(28,9 \%)$ a causa de insucesso foi rejeição.

\section{I S C U S S Ã O}

Nos últimos 50 anos, têm ocorrido alterações nas indicações de ceratoplastia penetrante em decorrência principalmente do desenvolvimento de materiais e técnicas cirúrgicas que cada vez mais aumentam o índice de sucesso e prognóstico cirúrgico ${ }^{(5,9)}$. A indicação do transplante de córnea varia de acordo com a epidemiologia e a prática do Serviço onde é realizado o procedimento $^{(1,5)}$. Em muitos Serviços, o ceratocone é a principal indicação para ceratoplastia penetrante ${ }^{(1,5,10)}$, embora outros evidenciem a ceratopatia bolhosa como indicação mais frequiente $^{(11)}$. Na presente amostra, o ceratocone e a ceratopatia bolhosa pós-facectomia foram as principais indicações para ceratoplastia penetrante, observando-se proporções semelhantes entre estes.

O glaucoma pós-ceratoplastia penetrante é um problema clínico significante devido à freqüência de ocorrência, dificuldade no reconhecimento e monitorização, e complexidade do tratamento $^{(2)}$. No presente estudo, assim como em outros estudos realizados ${ }^{(1)}$, o glaucoma foi a complicação pós-operatória mais freqüentemente encontrada, acometendo $31,7 \%$ dos pacientes. A presença e gravidade do glaucoma são fatores de risco conhecidos para a falência do transplante ${ }^{(1,12)}$. Neste estudo, o glaucoma esteve presente em $64,7 \%$ dos pacientes que evoluíram com falência tardia do enxerto. Postula-se que esta ocorra devido à perda endotelial secundária ao aumento da pressão intra-ocular ${ }^{(1,2)}$. Se o glaucoma permanece sem controle, pode levar à perda visual irreversível, devido à lesão progressiva do nervo óptico ${ }^{(2)}$. Observou-se neste estudo que entre os pacientes com AV final SPL, o glaucoma esteve relacionado no pós-operatório de $87,5 \%$ destes casos.

A ceratite infecciosa é uma complicação incomum e grave da ceratoplastia penetrante, levando à perda da transparência do enxerto em um grande número de pacientes ${ }^{(13)}$. No presente estudo, observou-se infecção pós-operatória do botão corneano em $6,3 \%$ do total de casos, incidência aumentada em relação à literatura que descreve variações de $1,76 \%$ a 4,9\% ${ }^{(13)}$. Este fato pode ser atribuído às diferenças epidemiológicas da região e ao baixo nível sócio-econômico da população estudada, o que dificulta o acesso do paciente à terapêutica indicada. Cerca de $77,0 \%$ dos pacientes que desenvolveram ceratite infecciosa no pós-operatório evoluíram com opacificação total do botão, retratando a gravidade desta complicação e suas conseqüências visuais devastadoras ${ }^{(13)}$.

As taxas de rejeição do enxerto corneano, dependendo da série estudada, variam de $3,5 \%$ a $65 \%$, de acordo com a vascularização da córnea receptora ${ }^{(7)}$. No presente estudo observou-se uma taxa total de rejeição de $24,4 \%$, havendo irreversibilidade da rejeição em $26,0 \%$, achado este inferior ao encontrado por outros autores $^{(7,10)}$. Acredita-se que reações leves, ao contrário das severas, não são seguidas de insucesso do transplante. A severidade da reação pode resultar numa diminuição da densidade de células endoteliais que excede a perda esperada e desencadeia a formação de opacidade permanente ${ }^{(1)}$. O glaucoma foi um dos principais fatores predisponentes à rejeição, acometendo $36,0 \%$ dos pacientes, dado que corrobora com a literatura mundial ${ }^{(3,6)}$. É importante que o cirurgião identifique previamente os fatores de risco presentes para a rejeição em cada caso a ser operado, e avalie se há baixo ou alto risco de rejeição do enxerto ${ }^{(2)}$.

O transplante de córnea realizado nos países em desenvolvimento tem apresentado pobres resultados da $\mathrm{AV}$, principalmente em categorias de pior prognóstico e na presença de algum fator de risco ${ }^{(9,14)}$. Pacientes de baixo nível sócio-econômico apresentam maior risco relativo para falência do transplante ${ }^{(14)}$. Neste estudo, os autores enfatizam as condições oculares pré-operatórias desfavoráveis encontradas em grande parte da população estudada, e a alta freqüência de pacientes com complicações oculares, que requerem cuidados terciários. É importante salientar que as cirurgias foram realizadas num serviço de referência, onde são admitidos casos mais complexos, e com 
maiores chances de complicações ${ }^{(1,3)}$. Maiores taxas de sucesso no transplante são descritas por cirurgiões mais experientes, o que pode ser explicado, em parte, pela maior experiência nos cuidados pós-operatórios, principalmente no diagnóstico precoce e tratamento das complicações ${ }^{(6,9)}$. Observou-se também uma maior incidência de glaucoma pré-operatório $(15,6 \%) \mathrm{em}$ relação a outros estudos ${ }^{(1)}$, o que agravou as complicações pós-operatórias.

Muitos fatores devem ser rigorosamente averiguados antes de submetermos o paciente a um transplante de córnea. Se houver história de dois ou mais procedimentos cirúrgicos além da ceratoplastia penetrante, glaucoma pré-operatório, sinéquias, neovasos ou aumento da pressão intra-ocular no pós-operatório, estes pacientes devem ser orientados e seguidos com maior frequiência com o intuito de se diagnosticar precocemente uma possível rejeição, e deste modo aumentar as chances de sucesso do transplante ${ }^{(3)}$.

A GRADECIMENTOS

À Professora Sylvia Lemos Hinrichsen e ao Professor Natal pela orientação metodológica e estatística.

\section{A B S T R A C T}

Purpose: To identify the main complications and causes of penetrating keratoplasty failure in interventions performed at Fundação Altino Ventura, Recife-PE. Methods: Medical records of 205 patients submitted to penetrating keratoplasty at the Fundação Altino Ventura, between January 1999 and December 2000, and with a follow-up after surgery of at least six months were revised. We analyzed demographic and other associated data from the pre, inter and post intervention periods. Results: The average age of patients was $48.4 \pm 22.1$ years, $108(52.7 \%)$ being female. The main indications for penetrating keratoplasty were keratoconus (49 cases, $23.9 \%$ ), bullous keratopathy after cataract extraction (48 cases, $23.4 \%$ ) and dystrophies ( 24 cases, $11.7 \%$ ). The most common postsurgery complication was glaucoma in 65 cases $(31.7 \%)$. Fifty patients $(24.4 \%)$ presented some clinical sign of rejection, leading to graft failure in 13 cases $(26.0 \%)$. It was observed that $16.0 \%(n=8)$ of the patients with graft rejection had been submitted to at least three interventions, which shows a sta- tistically significant difference from the group which showed no rejection $(3.2 \%, \mathrm{n}=5)(\mathrm{p}<0.05)$. Forty-five $(22.0 \%)$ cases developed corneal graft failure. Conclusions: The risk factors for graft failure identified in this study match those reported in the literature. Glaucoma was the main post-surgery complication and the main predisposing factor for rejection and graft failure.

Keywords: Keratoplasty penetrating/adverse effects; Postoperative complications; Treatment outcome

\section{REFER E N I A S}

1. Urbano AP, Akaishi PMS, Sarmento S, Urbano AP, Costa VP, Arieta CL. Evolução das ceratoplastias penetrantes realizadas por residentes. Rev Bras Oftalmol 2001;60:741-8.

2. O`Day DG. Glaucoma after penetrating keratoplasty. In: Krachmer JH, Mannis MJ, Holland EJ, editors. Cornea: Surgery of the cornea and conjunctiva. St. Louis: Mosby; 1997. p.1719-30.

3. Chalita MRC, Diazgranados EBM, Sato EH, Branco BC, Freitas D. Rejeição corneana pós-transplante de córnea: análise de dados do Banco de Olhos do Hospital São Paulo- Escola Paulista de Medicina. Arq Bras Oftalmol 2000; 63:55-8.

4. Fabris C, Corrêa ZMS, Marcon AS, Castro TN, Marcon IM, Pawlowski C. Estudo retrospectivo dos transplantes penetrantes de córnea da Santa Casa de Porto Alegre. Arq Bras Oftalmol 2001;64:449-53.

5. Dantas MCN, Dantas PEC, Holzchuh N, Lui Neto A, Giovedi Filho R, Giovedi M, Almeida GV. Indicações de transplante penetrante de córnea: 1991-1995. Arq Bras Oftamol 1998;61:26-33.

6. Vail A, Gore SM, Bradley BA, Easty DL, Rogers CA, Armitage WJ. Conclusions of the corneal transplant follow up study. Collaborating Surgeons. Br J Ophthalmol 1997;81:631-6.

7. Naacke HG, Borderie VM, Bourcier T, Touzeau O, Moldovan M, Laroche L. Outcome of Corneal transplantation rejection. Cornea 2001;20:350-3.

8. Alldredge OC, Krachmer JH. Clinical types of corneal transplant rejection. Their manifestations, frequency, preoperative correlates, and treatment. Arch Ophthalmol 1981;99:599-604.

9. Teixeira MF, Almeida Jr GC, Rodrigues ML, Kamimoto PS, Kashiwabuchi LK. Resultados e indicações de ceratoplastias penetrantes realizadas por médicos em treinamento, num país em desenvolvimento. Arq Bras Oftamol 2001; 64:557-61.

10. Williams KA, Muehlberg SM, Lewis RF, Coster DJ. How successful is corneal transplantation? A report from the Australian Corneal Graft Register. Eye 1995;9:219-27.

11. Patel NP, Kim T, Rapuano CJ, Cohen EJ, Laibson PR. Indications for and outcomes of repeat penetrating keratoplasty, 1989-1995. Ophthalmology 2000;107:719-24.

12. Kwon YH, Taylor JM, Hong S, Honkanen RA, Zimmerman MB, Alward WL, Sutphin JE. Long-term results of eyes with penetrating keratoplasty and glaucoma drainage tube implant. Ophthalmology 2001;108:272-8.

13. Santos NC, Sucomine PT, Sousa LB, Sato EH, Freitas D. Ceratite infecciosa pós-transplante de córnea. Arq Bras Oftalmol 1999;62:48-53.

14. Dandona L, Naduvilath TJ, Janarthanan M, Ragu K, Rao GN. Survival analysis and visual outcome in a large series of corneal transplants in India. Br J Ophthalmol 1997;81:726-31.

Nos artigos enviados para publicação, o nome dos autores e suas afiliações devem estar completos. Isso facilitará a indexação e os links com as bases de dados e o CV Lates. 